Published in final edited form as:

Theor Popul Biol. 2018 July ; 122: 97-109. doi:10.1016/j.tpb.2017.10.006.

\title{
Selecting among three basic fitness landscape models: additive, multiplicative and stickbreaking
}

\author{
Craig R. Miller ${ }^{a, b, c,{ }^{*}, \text { James T. Van Leuven }}{ }^{a}$, Holly A. Wichman ${ }^{a, b}$, and Paul Joyce ${ }^{c}$ \\ ${ }^{a}$ Center for Modeling Complex Interactions, University of Idaho, Moscow, Idaho 84844 \\ bDepartment of Biological Sciences, University of Idaho, Moscow, Idaho 83844 \\ 'Department of Mathematics, University of Idaho, Moscow, Idaho 83844
}

\begin{abstract}
Fitness landscapes map genotypes to organismal fitness. Their topographies depend on how mutational effects interact—epistasis—and are important for understanding evolutionary processes such as speciation, the rate of adaptation, the advantage of recombination, and the predictability versus stochasticity of evolution. The growing amount of data has made it possible to better test landscape models empirically. We argue that this endeavor will benefit from the development and use of meaningful basic models against which to compare more complex models. Here we develop statistical and computational methods for fitting fitness data from mutation combinatorial networks to three simple models: additive, multiplicative and stickbreaking. We employ a Bayesian framework for doing model selection. Using simulations, we demonstrate that our methods work and we explore their statistical performance: bias, error, and the power to discriminate among models. We then illustrate our approach and its flexibility by analyzing several previously published datasets. An R-package that implements our methods is available in the CRAN repository under the name Stickbreaker.
\end{abstract}

\section{Keywords}

fitness landscape; epistasis; additive; multiplicative; stickbreaking

\section{Introduction}

The fitness landscape is a modeling framework that maps DNA or protein sequence variants to fitness ([1], [2], [3], [4]). Adjacent locations on a plane represent genomes that differ by one mutational event. The fitness of each genotype is envisioned as forming a surface above the plane. Fisher's geometric model is closely related. There, the plane represents phenotype space (rather than sequence space) and again the surface above is fitness ([5], [3], [6], [7], [8]). In reality, of course, the genotype (or phenotype) plane is often highly dimensional; a two dimensional plane with a fitness surface above is used mainly because it begets the landscape metaphor and makes the model easier to conceptualize.

\footnotetext{
*Corresponding author: crmiller@uidaho.edu.
} 
Understanding the topography of the fitness landscape is important. It determines the extent to which recombination confers benefits, which bears on the potential advantages of sex ([9], [10], [11]); it has consequences for reproductive isolation as a mechanism for speciation ([12], [13], [14]); it dictates how stochastic versus predictable evolution is ([15], [16], [17], [18]); it plays a major role in how likely and at what speed adaptation is to find a highly optimal solution ([19], [20], [21]). But developing an understanding of real fitness landscapes is a serious challenge. First, the space is staggeringly vast and estimating its shape from a small sample of the space can be misleading ([22]). Even in a tiny viral genome of 5000 bases, there are $5000 \times 3=15,000$ possible first step DNA substitutions and the number of different genotypes with, say, just five mutations is on the order of $\left(\frac{5000}{5}\right) 3^{5} \approx 6 \times 10^{18}$. The number of unique pathways to each of these adds severals more orders of magnitude: $\prod_{k=1}^{5}\left(\frac{5}{k}\right)=2500$. Second, fitness on the landscape in real populations is rarely fixed; it shifts over time due to biotic and abiotic changes in the environment ([23], [24], [25], [26]). Third, the biology that underlies the fitness landscape may vary among systems and be complex, reflecting the fact that adaptation can occur in a multitude of ways (e.g., changing gene expression, protein stability, host-microbe interactions, metabolic pathways).

In the face of these challenges, researchers have pursued two major strategies to studying fitness landscapes: theoretical and empirical. An extensive body of theory has been developed that is based on various assumptions about relevant features such as the number and distribution of mutational effects on fitness, how mutations interact (epistasis), and the mutation-selection dynamics at work in the population (e.g. [27], [2], [23], [28], [29], [30], [6], [31], [20], [32], [33]). In the second approach, empirical data about the fitness landscape are collected ([4]). In microbial experimental evolution, a phenotype is measured that is expected to correlate strongly with fitness. Commonly used phenotypes include growth rate $([34,35,36])$, change in genotype frequency in an environment with competing genotypes $([37,38,39])$, or, for antibiotic resistance mutations, the minimum inhibitory concentration ([40, 41, 42]). Given the vast scope of sequence space, these studies have necessarily focused data collection on small regions of the landscape. One way to obtain an especially detailed view of the landscape is to begin with a small set of mutations, construct all combinations of them and then measure their fitness and/or phenotypes (e.g. [43], [44], [45], [16], [46], [47], [48], [49], [50], [51], [52], [53], [8]). In the landscape metaphor, this maps out all possible mutational pathways between the wildtype and the genotype with all mutations included. One common variation of this approach is to use pairs of mutations and engineer the two single mutants and double mutant genotypes (e.g. [54], [7], [55]); this amounts to creating many two-step, 4-genotype networks. As tools for genetic engineering improve, these experimental approaches are becoming increasingly feasible for greater numbers of mutations and larger mutational networks (e.g., [56], [57], [58]).

There is growing momentum in the field to bridge the theoretical and empirical ([4]). Much of mutational combination data has been fit or compared to models-either in the original work, in later analysis papers, or both. One common approach has been to assume a null model (usually the additive or multiplicative model) and characterize epistasis as deviations 
from this (e.g. [54], [47], [49], [55]). Another approach has been to characterize the extent of sign epistasis in the data (the case where mutations switch from being individually deleterious to being beneficial in combination, or vice versa) and, using a model of population dynamics, examine the probabilities of different pathways in the network (e.g. [43], [16], [59], [51], [53]). Other studies have fit the data to landscape models. Among explicitly fitted models, one group is based on mapping genotypes to fitness and includes the Rough Mt. Fuji model, the NK model, the uncorrelated model (also known as the House of Cards) as well as models more tailored to the biology of the study system (e.g. [44], [60], [50], [48], [61], [62], [63]). The other family of fitted models is based on Fisher's geometric model where mutations are assumed to have additive effects on phenotype and phenotypes then map to fitness (e.g. [45], [64], [7], [65], [26], [8]).

We believe that the endeavor of fitting data to landscape models can be strengthened by more carefully considering and developing simple models. More specifically, it has been generally overlooked that there are actually several equally simple fitness landscape models - additive, multiplicative, and stick-breaking ([66]) - any one of which can be taken as a basic model against which to compare more complex models. These models are similarly simple in that they all assume that fitness depends on the intrinsic effects of the constituent mutations. All three models lack higher order interactions ([67], [68]) or explicit phenotypic dimensions and thus have relatively few parameters to estimate. This benefit it not trivial because the amount of data available for model fitting is severely constrained: the full combinatoric network of $k$ mutations contains $2^{\mathrm{k}}-1$ observable effects. In the additive model, mutations have an absolute effect on the background fitness; in the multiplicative model their effect is proportional to the background fitness; in the stickbreaking model their effect is proportional to the distance between the background and the fitness optimum. At a biological level, the stickbreaking model emphasizes the existence of a local fitness optimum, and asserts that beneficial mutations will tend to have much larger effects when an organism is far from its optimum, but have diminished effects when near it. The multiplicative model, by contrast, asserts that beneficial effects tend to act synergistically, so that effect sizes become larger as a walk proceeds. The additive model assumes that effects are independent of background so that order does not change effect size. Both the additive and multiplicative model implicitly assume no fitness limit. While this is clearly unreasonable, we often focus in microbial evolution on a relatively small set of mutations; in this limited domain, a fitness boundary may or may not be relevant.

We argue that modeling always benefits from the existence and use of meaningful basic models. When basic models are rejected in favor of more complex ones, the rejection is more than a straw man; rather, the way the complex model differs from the simple models may offer insight into the underlying biology. For example, simple models could be rejected because there are multiple mutations that fix the same phenotypic problem or because one mutation is compensatory for another but deleterious when alone (i.e., sign epistasis). In other cases, a simple model may provide a good enough approximation to be useful. The purpose of this work is to develop the methods for fitting and comparing the three basic landscape fitness models to data. Using simulations and empirical data, we then illustrate how to use these methods. 


\section{Methods}

\subsection{Overview}

We begin by assuming that the data represent the complete set of $2^{k}$ genotypes created from $k$ mutations at different loci (wildtype included). Here we use the term wildtype to refer to the control or reference genotype and assume only two possible states at each locus. Later we return to the topic of other dataset structures. Our approach is to fit the data to each of three models where the models have the same structure: the observed fitness (or phenotype) of each genotype is the expected fitness (or phenotype) under the specified model plus Gaussian error. After establishing how to fit the three models, we develop methods to compare them and quantify model support using a Bayesian approach to assign posterior probabilities. Before delving in, we stress that the way fitness (or phenotype) is defined will affect the process of model fitting. For example, growth rate is an exponent and intrinsically occupies that scale while the number of offspring occupies the base or arithmetic scale. Thus, additivity of growth rate effects is equivalent to multiplicative effects on the absolute number of offspring. Sailer and Harms ([68]) show how epistasis can emerge as a consequence of a mismatch between the scale on which mutations interact and the scale their effects are measured on. A similar concern is that the way mutations are discovered for inclusion in these networks can influence the nature of their epistatic interactions ([22], [69]). One of the advantages of our approach here is that it makes no a priori assumptions about which model is most appropriate (among additive, multiplicative, or stickbreaking). Nonetheless, it remains important for researchers be aware of the role of scale, the source of mutations under study, and of the way fitness definition plays into model selection, and to interpret their results accordingly.

\subsection{Notation}

We begin by establishing some notation, much of which is standard. We will use capital letters to denote sets of mutations or random variables. It should be clear from the context whether we are referring to a set of mutations or a quantity which is observed with error (random variable). Let $K=\{1,2, \ldots, k\}$ be the set of all mutations under study. Let the set of mutations comprising a genotype be denoted by $G$, where $G$ is a subset of $K(G \subset K)$ on the wildtype background. We will use small letters to represent elements within a set, or parameters of the model. For example we may refer to mutation $i \in G$ as a single mutation among those in the set of mutations denoted by $G$.

\subsection{Basic models}

If there were no errors or noise in the model, then under the additive model, the fitness of genotype $G, w_{G}$, would be

$$
w_{G}=w_{w t}+\sum_{i \in G} \Delta w_{i}
$$

where $\Delta w_{i}$ is the intrinsic effect of mutation $i$ and $w_{w t}$ is the fitness of the wildtype. Fitness under the multiplicative model would be 


$$
w_{G}=w_{w t} \prod_{i \in G}\left(1+s_{i}\right)
$$

where $s_{i}$ is the intrinsic selection coefficient of mutation $i$.

In the stickbreaking model, the effect of a mutation is to close the distance to the fitness optimum by a proportion specified by its coefficient ([66]). Thus, when a mutation has a stickbreaking coefficient of 0.25 , it moves fitness $25 \%$ of the way to the fitness optimum. If the same mutation occurs on backgrounds of increasing fitness, the absolute effect of the mutation will diminish. Formally, the expected fitness under the stickbreaking model is given by

$$
w_{G}=w_{w t}+d\left(1-\prod_{i \in G}\left(1-u_{i}\right)\right)
$$

where $u_{i}$ is the intrinsic stickbreaking coefficient of mutation $i$ and $d$ is the fitness difference between the fitness boundary and the wildtype (see [66] for derivation of the stickbreaking model).

Even when one of these models is valid, we expect real data to deviate from the expected values for two reasons. First, the models are, at best, approximations of reality and deviations due to the underlying biological processes will exist. Second, there is experimental error in real data. We accommodate both of these sources of noise by combining them into one term such that the observed fitness of any genotype is given by its predicted fitness under the model plus a normally distributed error: $W_{G}=W_{G}+\varepsilon$ where $\varepsilon \sim$ $\mathrm{N}\left(0, \sigma^{2}\right)$ and $w_{G}$ is given by equations (1), (2), and (3). We assume that the errors are independent across genotypes. Note that the stickbreaking and multiplicative models involve products instead of sums. This means they reside naturally on the log scale while the additive model is on the non-log scale. It might seem appropriate, therefore, to model errors for stickbreaking and multiplicative as log-normal (normal on the log scale). However, experimental error does not depend on the underlying model and using different models of error makes comparison across models difficult. Finally, using normal, and not log-normal, error allows us to use a maximum likelihood estimate of $d$. Estimation of $d$ in the stickbreaking model is taken up in the next subsection. This is followed by subsections, applicable to all three models, on estimating coefficients, estimating $\sigma$, and doing model selection.

\subsection{Estimating distance to the fitness boundary, $d$, under stickbreaking}

The first step in fitting the stickbreaking model to real data (which we do in the next subsection) is to estimate $d$. We develop three different methods of estimation. 
Method 1: Maximum likelihood-Because we are assuming error is normally distributed, the maximum likelihood estimate (MLE) will be that value of $d$ that minimizes the squared differences between observed fitness and the predicted fitness (right side of 3):

$$
\hat{d}_{\mathrm{MLE}}=\min _{G}\left(\left[w_{G}-w_{w t}-d\left(1-\prod_{i \in G}\left(1-u_{i}\right)\right)\right]^{2}\right) .
$$

In practice, we find the MLE using the optimize function in R. All of the computational work in this paper is done the $\mathrm{R}$ environment ([70]).

Method 2: Relative Distance to Boundary (RDB) estimator-Equation (3) can be rewritten as

$$
d=\frac{w_{G}-w_{w t}}{1-\prod_{i \in G}\left(1-u_{i}\right)} .
$$

Notice that with perfect knowledge of the coefficients and in the absence of noise, any genotype $G$ on the right-hand side of (5) could be used to calculate $d$. The strategy is to begin by estimating $\Pi_{i \in G}\left(1-\mathrm{u}_{i}\right)$ and then use that estimate in (5) to estimate $d$. The expression $\Pi_{i \in G}\left(1-u_{i}\right)$ represents the relative distance to the boundary (RDB) for genotype $G$. If genotype $G$ produces a fitness gain of $W_{G}-W_{w t}$ then the distance to the boundary would be $d-\left(w_{G}-W_{w}\right)$ and the relative distance to the boundary would be $r_{G}=\frac{d-\left(w_{G}-w_{w t}\right)}{d}$. So it follows from (3) that

$$
r_{G}=\frac{d-\left(w_{G}-w_{w t}\right)}{d}=\prod_{i \in G}\left(1-u_{i}\right) .
$$

It would appear from equation (6) that in order to calculate the relative distance to the boundary $r_{G}$ using observable fitness effects, one would need to know $d$ a priori. However, in the APPENDIX we obtain an expression for $r_{G}$ based on observable fitness effects independent of $d$. Equation (18) in the APPENDIX shows that

$$
r_{G}=\frac{{ }^{w_{K}}-{ }^{w} G}{w_{G}{ }^{-w_{w t}}}=\prod_{i \in G}\left(1-u_{i}\right) .
$$

This leads to the following estimate $\hat{r}_{G}$ for the RDB of $G$

$$
\widehat{r}_{G}=\frac{W_{K}-W_{G}}{W_{G}^{c}-W_{w t}}
$$


which leads to a set of estimates for the boundary $d$ given by

$$
\widehat{d}_{G}=\frac{W_{G}-W_{w t}}{1-\hat{r}_{G}} .
$$

We now define the set of all estimates of $d$ given by (8) to be

$$
D=\left\{\hat{d}_{G}: G \subset K\right\} .
$$

$D$ can be viewed as a transformation of the fitness effect data. $D$ contains $2^{k}-1$ estimates of $d$. A measure of the center of the transformed data $D$ will form our final estimate of $d$. Both the average and median of $D$ produce valid estimates of the distance to the boundary $d$. We did extensive simulations on the properties of the mean versus the median; based on both bias and root mean squared error we conclude that, for the noise levels explored here, the median estimator is the better alternative. Thus, this RDB estimator, which uses all genotypes, is

$$
\hat{d}_{\mathrm{RDB}_{\mathrm{all}}}=\operatorname{median}(D) .
$$

Notice that for data without error, $r_{G}$ can only fall between 0 and 1 . With noise, however, genotypes can generate values outside this range. In particular, genotypes where $W_{G}<W_{W t}$ or $w_{G}>w_{k}$ cause problems. This led us to consider a modification to the RDB estimator where we use only the subset of $D$ that come from genotypes where $0<r_{G}<1$, denoted $D_{01}$. In the results section we will show that this modified estimator outperforms $\hat{d}_{\mathrm{RDB}_{\text {all }}}$. For notational simplicity we hereafter denote it as just $\hat{d}_{\mathrm{RDB}}$. Formally then, the estimator is,

$$
\hat{d}_{\mathrm{RDB}}=\operatorname{median}\left(D_{01}\right) \text {. }
$$

Method 3: Hybrid estimator-In order to do model selection (below), it is invaluable if we can fit the stickbreaking model to every dataset, even if the fit is very poor. The two estimators just described do not always produce valid estimates of $d$ and this prohibits fitting the stickbreaking model to every dataset. We define a valid estimate to be $d>0$ and $d<$ $10\left(W_{\max }-W_{W t}\right)$ where $W_{\max }$ is the maximum observed fitness. An estimate $<0$ implies a fitness boundary lower than the wildtype fitness. The reason for not accepting values more than ten times the largest fitness difference from wildtype (i.e. $10\left(w_{\max }-w_{W t}\right)$ ), is because we want the stickbreaking model to be distinct from the additive model, yet the stickbreaking model approaches the additive model as $d$ gets large and the coefficients get small ([66]). 
What can be done when the MLE and RDB estimators both fail? One guaranteed way to always obtain an estimate of $d$ is simply to use a value slightly larger (say 10\%) than the largest observed fitness: $\hat{d}_{\operatorname{Max}}=\left(\max \left(w_{i}\right)-w_{w t}\right)(1.1)$ for all $i \subset G$. Note that defining $d$ this way results in maximal coefficient estimates and generally a poor fit to the data. Our view is that if the data is so noisy and problematic that we cannot obtain a good estimate of $d$, then it is appropriate to disfavor the stickbreaking model by using a small estimate of $d$. We suggest, then, the following rule as a way to estimate $d$ across all datasets: use $\hat{d}_{\text {MLE }}$ unless it fails to produce a meaningful estimate; use $\hat{d}_{\text {RDB }}$ unless it fails; then use $\hat{d}_{\text {Max }}$. We justify this order in the results section below. We refer to this procedure for estimating $d$ as $\hat{d}_{\text {hybrid }}$.

\subsection{Estimating coefficients}

When mutation $i$ is added to background $B$ (where $i$ is not in $B$ ), denote this genotype $B^{i}$. Under the additive model, the expected value $E\left(W_{B^{i}}\right)=w_{B}+\Delta w_{i}$, and hence a natural way to estimate the coefficient for mutation $i$ is just $\Delta \widehat{w}_{i}=W_{B}{ }_{B}-W_{B}$ and average over all $B$ and $i$.

We make a small adjustment to this by weighting the observations on the wildtype background twice as heavily as the other genotypes. This is because we assume wildtype will generally serve as a control in fitness estimation with the consequence that it is observed more times and thus estimated much more precisely than the other genotypes. The consequence is that the variance of the difference $W_{B^{i}}-W_{B}$ will be $\sigma^{2}$ when $B$ is wildtype, and $2 \sigma^{2}$ when $B$ is not wildtype. Weighting by the inverse of the variance, we get,

$$
\Delta \widehat{w}_{i}=\frac{W_{B_{j}^{i}}-W_{w t}}{2(m+1)}+\sum_{j \in B^{i} B \neq w t} \frac{W_{B_{j}^{i}}-W_{B}}{m+1}
$$

where $j \in B^{i}$ indicates the sum over all genotypes containing $i, B \neq w t$ means exclude the case where $B$ is wildtype, and $m$ is the number of backgrounds on which $i$ appears ( $m=2^{k-1}$ or half the genotypes in the dataset).

Because the multiplicative model involves a product, it is simplest on the log-scale. Taking the log of both sides of equation (2) and defining $y_{G}$ as the transformed fitness, we have

$$
y_{G}=\log \left(w_{G}\right)-\log \left(w_{w t}\right)=\sum_{i \in G} \log \left(1+s_{i}\right) .
$$

If we let $y_{i}=\log \left(1+s_{i}\right)$, then equation 13 implies that $E\left(Y_{B}{ }_{B}\right)=y_{B}+y_{i} \cdot{ }_{B^{i}}-Y_{B}$ therefore provides an estimate of $y_{i}$. We weight the estimates according to whether one or both backgrounds are observed with error and then transform back to the non-log scale: 


$$
\hat{s}_{i}=\exp \left(\frac{Y_{B_{j}^{i}}-Y_{w t}}{m+1}+\sum_{j \in B^{i} B \neq w t} \frac{Y_{B_{j}^{i}}-Y_{B}}{2(m+1)}\right)-1 .
$$

For stickbreaking, we also transform to the log scale by taking the log of equation (3), rearranging, and defining $z_{G}$ as the transformed fitness,

$$
z_{G}=\log \left(1-\frac{w_{G}-w_{w t}}{d}\right)=\sum_{i \in G} \log \left(1-u_{i}\right) .
$$

Letting $Z_{i}=\log \left(1-u_{i}\right)$ and replacing $d$ with $\hat{d}$ in equation (15), we see that $E\left(Z_{B}{ }^{i}\right)=z_{B}+z_{i}$ so that $Z_{B}{ }_{B}-Z_{B}$ provides an estimate of $Z_{i}$. Again, we estimate $i$ over all genotypes it appears in, weight by the inverse of the variance and then transform the estimate back to the non-log scale,

$$
\hat{u}_{i}=1-\exp \left(\frac{Z_{B_{j}^{i}}-Z_{w t}}{m+1}+\sum_{j \in B^{i} B \neq w t} \frac{Z_{B_{j}^{i}}-Z_{B}}{2(m+1)}\right) .
$$

\subsection{Estimating $\sigma^{2}$}

Recall that we assume all genotypes in the dataset except wildtype depart from their predicted value as independent random normal deviates with mean 0 and variance $\sigma^{2}$. We thus estimate $\sigma^{2}$ by,

$$
\hat{\sigma}^{2}=\sum_{G=2}^{T} \frac{\left(W_{G_{(o b s)}}-W_{G_{(p r e d)}}\right)^{2}}{T-2}
$$

where $W_{G_{(p r e d)}}$ comes from substituting the estimated coefficients (and $\hat{d}$ in the case of stickbreaking) into the appropriate equation (equation (1), (2) or (3)).

\subsection{Assessing fit and model selection}

We are ultimately interested in determining which of the models (stickbreaking, multiplicative, or additive) are consistent with a set of data. Because it is straightforward to calculate the likelihood of the data under the three models, we first pursued using AIC to do model selection. To our surprise, this approach was unsuccessful. When we analyzed simulated data, we observed that under parametric conditions with low signal to noise ratios, the true model was falsely rejected an unacceptably high fraction of the time (i.e. $\geq 5 \%$ ). We 
believe this owes to the nonstandard nature of the data as a network where each observation involves a different subset of parameters. We eventually abandoned AIC. Instead, we developed a Bayesian approach that creates a predictive model of posterior probability by training it on simulated data. The method has four steps: (i) simulate data from priors, (ii) fit data to each model, estimate parameters, and generate summary statistics, (iii) feed the summary statistics into a multinomial regression to train it, and (iv) use the multinomial regression model on other data (e.g. real data) to calculate the probability it comes from each of the three models.

We now cover these four steps (denoted i-iv) in greater detail. In step (i), we do simulations. We conduct separate simulations for networks with 3, 4, and 5 mutations. For each number of mutations, we simulate 10,000 datasets by drawing parameters from uniform prior distributions: each model (stickbreaking, multiplicative, additive) has equal (1/3) probability, a coefficient value ( $u, s$, or $\Delta w$ depending on the model) is sampled from a uniform $(0.05$, $0.5)$, this value is assigned to all mutations in the dataset, and $\sigma$ is sampled from a uniform $(0.01,0.1)$. For stickbreaking datasets, $d=1$ throughout. We then simulate datasets according to the assumptions described in the 'Basic Models' section above.

In step (ii), we fit the data to each model. For stickbreaking, this requires first estimating $d$. For all three models, we then estimate the coefficients for each mutation. (Note, while we use the same coefficient value across mutations when simulating data, we estimate each one individually during the analysis.) We then summarize the fit using two statistics for each model: $R^{2}$ and a P-score. $R^{2}$ gives an overall measure of how close the predicted fitnesses are to the observed values under each model. It is obtained by calculating the mean fitness over the network (excluding wildtype), taking the squared deviations from this mean, and summing to get the total sum of squares (TSS). We then estimate the coefficients (and $d$ in the case of stickbreaking) and plug these estimates into equations (1), (2), and (3) to get predicted fitness values for every genotype in the network. We next take the differences between the predicted values and observed values, square them, and sum to get the residual sum of squares (RSS). Then, $R^{2}=1-R S S / T S S$. Note that this is not regression and there is no guarantee that the predicted values will be closer to the observed values on average than the overall mean is. Thus, it is possible for poorly fitting models to generate $R^{2}$ values $<0$.

While $R^{2}$ examines the nearness of observed and predicted values, the P-score assesses whether the pattern of deviations is consistent with a model. In short, if the data arose under the model being considered, then the observed fitness effects (as defined under that model) should not show a trend with increasing background fitness. If the data arose under a different model, they should should show a non-zero trend. To make this more precise, consider first the additive model. Let $B^{i}$ and $B$ be a background with and without mutation $i$. By equation (1), $W_{B^{i}}-W_{B}$ has expected value $\Delta W_{i}$ regardless of what background is considered. Thus, if the data arose under the additive model and we regress ${ }_{B^{i}}{ }^{i} W_{B}$ against $W_{B}$ for all $B$, we expect a line with intercept $\Delta w_{i}$ and slope zero. If the data instead arose under the multiplicative or stickbreaking models, we expect positive and negative slopes, respectively, when we do this regression ([66]). The analogous argument for the multiplicative model leads to the conclusion that if the data arose under it, regressing 
$Y_{B}{ }_{B}-Y_{B}$ against $W_{B}$ should yield a zero slope. If the data arose under the stickbreaking

model, it is the regression of $Z_{B^{i}}-Z_{B}$ against $W_{B}$ that should show no slope. Note that this is the same approach pursued by Khan et al. ([49]), except that they only considered the additive model.

Our linear regression test to generate a P-score is, therefore, to take each mutation $i=1,2$, $\ldots, k$, consider each background upon which it appears, calculate the observed effect under each of the three models ( $W_{B}{ }^{i}-W_{B}$ for additive, $Y_{B}{ }_{B}-Y_{B}$ for multiplicative, and $Z_{B}{ }_{B}-Z_{B}$ for stickbreaking), and regress these against $W_{B}$. We then fit these data points to a simple linear model using least-squares and obtain a p-value. The information in the p-values $\left(p_{1}\right.$, $\left.p_{2}, \ldots, p_{N}\right)$ are then summarized by taking the sum of the logs of the p-values to yield a Pscore: $P=\sum_{i=1}^{N} \ln \left(p_{i}\right)$. The smaller the p-values across mutations, the more negative the $\mathrm{P}$ score becomes. Notice that the pattern of departure from zero under the incorrect model is not actually linear ([66]). By assuming it is linear, we forego some power but benefit in terms of simplicity and computational speed.

Upon completing step (ii), the results are summarized as a matrix of 10,000 rows (one for each datset) by seven columns, one for the true model and six for the fit statistics: $R_{\text {stick}}^{2}$, $R_{\text {mult }}^{2}, R_{\text {add }}^{2}, P_{\text {stick }}, P_{\text {mult }}$ and $P_{\text {add }}$. In step (iii), we use the matrix of results to do multinomial regression using the neural networks package in $\mathrm{R}$, nnet. The multinomial regression uses six predictor variables ( $R_{\text {stick }}^{2}, R_{\text {mult }}^{2}, R_{\text {add }}^{2}, P_{\text {stick }}, P_{\text {mult }}$ and $P_{\text {add }}$ ) to calculate the probability the dataset arose under each of the three models (stickbreaking, multiplicative, and additive). (Preliminary exploratory work using different combinations of summary statistics indicated that these six are a very good set for doing model selection). Regression is done separately for 3,4 , and 5. Once the model has been trained, it is ready to use on other datasets (step iv). To do so, a dataset is fit and summarized (step ii) and the summary statistics are passed into the previously trained multinomial regression model from step (iii) to yield posterior probabilities.

\subsection{Incomplete networks}

Not all datasets contain the entire network with all $2^{k}$ genotypes formed from $k$ mutations. One instance of this is simply when individual genotypes are missing from the network. Another case (which we refer to as a double-mutant set) is when the network contains just four genotypes: wildtype, two individual mutations, and their combination double mutant. Suppose there are multiple such double-mutant sets. One possibility is that the mutations in each set are different (i.e. no mutations are shared). This generates an identifiabiltiy problem for stickbreaking (four datapoints yield three observed effects and there are three stickbreaking parameters to estimate) and we do not attempt to fit such data. Alternatively, it is possible that the same mutations appear across multiple double-mutant sets. In this case, we can view the data as a sample of the first and second steps of the much larger network. For example, in a bacteriophage dataset that we will analyze later, nine single mutations 
were engineered in various combinations to generate 18 double mutants. We think of this as 28 genotypes (including wildtype) of the full 512 genotype network $\left(2^{9}=512\right)$.

Whenever the network is incomplete, a few minor adjustments to our approach are necessary. First, recall that the RDB estimator of $d$ requires pairing a genotype with its complement (i.e. genotypes with and without a set of mutations). With incomplete sets, the necessary genotypes are often absent. When we cannot get RDB estimates of $d$ for each mutation, we cannot employ this estimator. In this case, our approach is to use the MLE method if it provides a valid estimate, and largest observed fitness if it does not. Second, recall our method of model selection entails $P$-scores that are based on regressing effect size against background fitness. Linear regression requires three datapoints. If we do not have a mutation on three backgrounds, we cannot perform the linear regression. Thus, sometimes one or more mutations will fail to yield p-values. Our approach is to base the $P$-score on the mutations we do get p-values from. If we cannot get any $\mathrm{p}$-values, we do model selection using $R^{2}$ values alone. This approach is justified by the next adjustment. Third, we must rerun the model training simulations where we sample 10,000 datasets from our priors, but instead of using the full network as before, we use whatever data structure is observed in the real data. As before, we then fit each dataset to each model and then use multinomial regression to train a model for assigning posterior probabilities to the three models.

One word of caution about incomplete datasets is warranted. If a genotype is absent because it is inviable, then omitting it will bias the analysis. While one could assign such samples a fitness of zero, this will also introduce bias because, in reality, inviable genotypes represent a boundary condition that the models fail to incorporate.

\section{Results and Discussion}

The goal of this work is to establish a robust statistical framework for fitting basic models of epistasis to empirical data. To do this, we first need to fit the additive, multiplicative, and stickbreaking models to the data and, second, do model selection. For the additive and multipicative models, fitting is straightforward but for stickbreaking the distance parameter, $d$, must first be estimated. We therefore open with a subsection on estimating $d$ and proceed to one on fitting data (i.e. estimating coefficients), then to model selection and finally to several subsections that deal with the analysis of different types of real data.

\subsection{Estimation of $d$}

To determine the best method of estimation, we simulated data under the stickbreaking model, setting $d=1$, considering effect sizes that ranged from $u=0.1$ to 0.5 in 0.1 increments, noise levels that ranged from $\sigma=0.02$ to 0.1 in 0.02 increments, and complete genotype networks comprised of 3,4 , or 5 mutations. The results for a subset of illustrative cases with 3 or 4 mutations are presented in Figure 1. The MLE estimator fails most often, but when it does work, it is generally the least biased and has error at or below the others. Thus, it is the estimator of first choice in the hybrid method. Between the two RDB (relative distance to boundary) estimators, the one that uses the subset of genotypes with an estimated boundary in the interval 0 to 1 ( $\hat{d}_{\mathrm{RDB}}$; in black) outperforms the one that uses all genotypes 
( $\widehat{d}_{\mathrm{RDB}_{\text {all }}}$; in grey). It fails far less frequently, tends to be less biased, and has similar rMSE

(root mean squared error); thus it is the second choice. The estimator based on the observed maximum fitness, $\hat{d}_{\text {Max }}$, by definition never fails, but it is chronically biased low. It is the estimator of last resort. All estimators become good as signal to noise improves. The inset pie charts show that the hybrid estimator is dominated by the MLE and RDB estimators with the Max estimator only appearing when signal to noise ratio is very poor.

\subsection{Coefficient estimation}

Each of the three models has coefficients associated with each mutation that we estimate from the data. For stickbreaking, $d$ is estimated first and then the coefficients are estimated based on $\hat{d}$. Figure 2 shows the rMSE and bias for the stickbreaking coefficients based estimates of $d$ from the hybrid method. The figure demonstrates three things. First, error and bias in estimates of $d$ leads to substantial error and some bias in estimating $u$. Second, small effect sizes are associated with large proportional error: at $u=0.1$ the rMSE is also around 0.1 . The errors as a proportion of the effect size are much smaller for $u=0.3$ and $u=0.5$ where relative errors are more on the order of $1 / 3$ and $1 / 5$ respectively. Third, reducing noise (i.e. decreasing $\sigma$ ) has a greater effect than increasing the number of mutations. For example, when we hold $u$ at 0.3 and compare 3 mutations and $\sigma=0.02$ (small network, low noise) with 5 mutations and $\sigma=0.08$ (large network, high noise) we see respective rMSE values of 0.037 and 0.063 . Said another way, if there is a choice between generating larger networks versus reducing noise, reducing noise is the more effective way of getting good parameter estimates. Of course, if the noise is not experimental but biological, then it cannot be reduced. In comparing models, coefficient estimates under the additive and multipicative models have much smaller errors (Figure 3). Our simulations also confirmed that estimates under the multiplicative and additive models are unbiased (results not shown). This disparity between stickbreaking and the other models comes from the fact that the stickbreaking coefficients depend on estimating another parameter first while multiplicative and additive coefficients do not.

\subsection{Model selection}

We are ultimately interested in identifying which of the three models best explains a given dataset. We took a Bayesian strategy for model selection in which we simulated a large number of datasets by sampling from prior distributions. Each dataset was then fit to each of the three models and summarized using $R^{2}$ and a linear regression generated P-score (see Methods). We then passed these six measures of fit and the true model's identity to a multinomial regression and allowed it to build a model that predicts the true model from the measures of fit. We did this for networks involving 3, 4, and 5 mutations separately. The coefficients from this multinomial regression are presented in Table 1. We next generated test data. To do this we gridded parameter space: 3 true models (stickbreaking, multiplicative, additive $) \times 3$ network sizes $(3,4,5$ mutations $) \times 5$ coefficient values $(0.1,0.2$, $0.3,0.4,0.5)$, and $4 \sigma$ values $(0.02,0.04,0.06,0.08)$. We then simulated 100 datasets per parameter combination. The performance of model selection was summarized as the mean posterior probability assigned to the true model and the proportion of replicates where the true model was rejected by having $<5 \%$ posterior probability. 
This model selection method did a good job of limiting false rejections of the true model (type 1 errors). Of the 180 conditions tested, 175 of them had five or fewer false rejections in the 100 replicates. The remaining instances were scattered in parameters space and, among these, error rates were not beyond what we would expect given a sample size of 100: two instances of 8 false rejections, two of 7 false rejections, and one of 6 false rejections. The other critical part of model selection is how often it hones in on the true model and rejects others. Figure 4 shows the mean posterior probability of the true model under all 180 parameter conditions we studied. Surface regions in white highlight parameter space where the true model has posterior probability $295 \%$ while darker grey regions are those with lower posterior probability. White regions with high posterior probability correlate very closely with regions where the true model is uniquely identified a high frequency of the time. Two main trends jump out of these results. First, model selection is hard with only three mutations, better at four, and a lot better with five mutations. Stated more precisely, model selection leads to uniquely identifying the true model over a much greater range of parameter space as the number of mutations increases.

The second major pattern in the results is that the multiplicative model is the easiest to identify when true followed by stickbreaking and then additive. The multiplicative model ranks first because it produces the most distinct data: effect sizes for each beneficial mutation increase as background mutations accumulate even though (under our model) the error associated with them stays on the same scale. The stickbreaking model is opposite in that effect sizes shrink with accumulating mutations. While this leads to a distinct expected pattern in the data, two features of stickbreaking complicate things. One is the fact that the distance to the boundary, $d$, must be estimated from the data (unlike the other models). The other is the fact that while effects are shrinking with accumulated mutations, the error around them is not. The additive model ranks third simply because it produces patterns intermediate between the other two models. While data from the stickbreaking model is very rarely confused with the multipicative model and vice versa, data from the additive model can, by chance, resemble either stickbreaking or multiplicative.

\subsection{Robustness to model assumptions}

Our method assumes the errors associated with all genotypes (except wildtype) come from the same normal distribution and when we simulate training datasets to fit in the multiple regression model, all coefficients are assumed to be the equal (although our analysis of data allows coefficients to vary). Furthermore, the evaluation of our methods (i.e. the above subsections on estimation of $d$, coefficient estimation, and model selection) was done by simulating datasets with equal coefficient values and errors drawn from a single distribution. We tested how robust our methods are to these assumptions by simulating data where coefficients were heterogeneous across mutations, where $\sigma$ was heterogeneous across genotypes, or both coefficients and $\sigma$ were heterogeneous, but analyzed these data with our standard approach and standard assumptions. We ran simulations across a gradient of increasing variability in both parameters (coefficients and $\sigma$ ) while maintaining the same mean values for them, and repeated the exercise at different mean values (low and high). For $\sigma$ we explored both correlated structures where increasingly fit genotypes had lower (or 
higher) $\sigma$ and randomly assigned the same values across genotypes. We assessed how this heterogeneity ultimately effects model selection.

Our results revealed that our methods are very robust to heterogeneity; the effect of adding this variability is to modestly lower the power to identify the true model (detailed results not shown). In no cases did we see the rate of false rejections rise above 5\%. For coefficient heterogeneity, we found that the posterior mean of the true model was always highest when coefficients were identical and was reduced modestly as coefficient variability increased. For $\sigma$ we found a similar, but weaker, effect of increasing heterogeneity. Thus, when real data contains variable effect sizes and errors across genotypes do not come from a single distribution, our method is conservative in that it simply becomes less confident which model is correct.

\subsection{Analysis of Real Data}

To illustrate how our method may be implemented we selected several datasets from the literature. The first is from a study on fitness recovery in a Methylobacterium engineered with a foreign metabolic pathway that it must employ to grow on the sole carbon source of methanol ([48]). Nine mutations were identified over the course of adaptation. Four of these mutations were engineered in all combinations to form the complete 16 genotype network. The data fitted to each of the three models is shown in Figure 5A. When passed to the multinomial regression model, $99.1 \%$ of the posterior probability is assigned to the additive model. In their paper, Chou et al. ([48]) developed an elegant cost-benefit model of the underlying metabolic processes, measured relevant phenotypes, and obtained a very good fit to the data. While their model provides more biological insight, the additive model gives a very good approximation of the fitness effects observed among their mutations.

The second dataset we analyzed was an experiment by Kahn et al. ([49]). Here the first five beneficial mutations in a long-term adaptation of Escherichia coli were engineered on the ancestral background in all 32 possible combinations. In their analysis, Kahn et al. examined additive fitness effects for each mutation as a function of background fitness. They showed that three of the five mutations in their dataset showed decreasing effects, one was not significantly different from zero, while one showed an increasing trend. These patterns correspond to our expectations under stickbreaking, additive, and the multiplicative models, respectively. Not surprisingly, when we analyzed the full 32 genotype network, we got ambiguous results with posterior probabilities for stickbreaking, multiplicative, and additive being $0.22,0.40$, and 0.38 . We then removed the one strongly multiplicative mutation (+pykF) and reanalyzed the 16 genotype network. When we did this we found that the data favors the strickbreaking model with 0.86 posterior probability compared to 0.10 for additive, and 0.04 for multiplicative. The fit of the data to the three models is illustrated in Figure 5B. Kahn et al. close their paper by stating that their results suggest a relatively simple epistasis function might be incorporated into models seeking to predict adaptation, though mutation $+\mathrm{phkF}$ demonstrates that there will be exceptions. Our results suggest that the stickbreaking model could provide exactly this type of simple function for approximating a common type of epistasis during adaptation. 


\subsection{Analysis of partial network data}

Up to this point we have assumed the data covers all possible combinations of the studied mutations. However, this will not always be the case. In some instances there will be missing genotypes in the network. Another common type of dataset involves single mutants examined alone and in combination as the double mutant. If the same mutations are used across multiple double-mutant sets, then such data can be fit to the three models. A good example of this comes from work by Caudle et al. ([26]) on the bacteriophage ID11. Here, nine first-step beneficial mutations that arose during replicate adaptations at $37^{\circ} \mathrm{C}$ were engineered into 18 of the possible 72 double mutants; none of the higher order genotypes (eg. triples, quadruples, etc.) were created. Fitness was estimated at 33,37 and $41^{\circ} \mathrm{C}$. The datasets at 33 and $37^{\circ} \mathrm{C}$ show such extensive sign epistasis that they do not fit any of the models considered here at all well (i.e. $R^{2}$ values are $<0$ ). At $41^{\circ} \mathrm{C}$, however, sign epistasis was more moderate appearing in 7 of the 18 doubles, but being reciprocal (where both first steps are deleterious on the background of the other) in only two cases. When we fit the $41{ }^{\circ} \mathrm{C}$ data to the three models, we find that the stickbreaking model does a much better job than the others (Figure 6A). When the $R^{2}$ and P-scores are passed to the multinomial regression model, $99.8 \%$ of the posterior probability is assigned to the stickbreaking model. This is not to argue that stickbreaking is the best possible model here. Caudle et al. were able to achieve a considerably better fit to this data $\left(R^{2}=0.55\right.$ and 0.82$)$ using more complex models that, in this case, involved gamma-shaped phenotype-fitness functions. Nonetheless, this analysis illustrates that our approach can be used on this type of dataset that features only single and double mutants so long as sign-epistasis is rare.

\subsection{Analysis of deleterious data}

Perhaps non-intuitively, our methods can also be used to analyze deleterious mutations, or even combinations of beneficial and deleterious mutations. We illustrate this by analyzing attenuation data from poliovirus. Burns et al. ([71])) recoded four contiguous capsid coding regions of 171-262 residues in length with synonymous mutations representing less preferred codons. They then created 10 of the possible 16 combinations of the recoded blocks and measured viral yield (plaque forming units or PFUs) over a $12 \mathrm{~h}$ growth period. We fit their data to the three models after log-transforming PFUs (since when growth is exponential, growth rate is proportional to the log of population size). The results, presented in Figure 6B, indicate that the additive model best describes the data, receiving over $99 \%$ of the posterior probability. This result is consistent with a result from the original paper where they found a strong, negative linear relationship between number of sites modified and PFUs.

\section{Conclusion}

We close with a few words about limitations and potential extensions of the framework advanced here. In terms of limitations, we have combined biological variance and experimental noise into a single variance term; in reality, variance may differ among genotypes and there is generally information about how much of the noise is experimental (versus biological) based on variance observed across replicates. This complexity could be added to our model in the future. Another limitation is that the interactions among all 
mutations are governed by the same model. Depending on the genes and mutations involved, this assumption may be violated (e.g. [49]). We experimented with developing a block version of our model motivated by Orr ([72]) where mutations are grouped into blocks and mutations in the same block share the same model of epistasis. We ultimately ran into an overfitting problem, but if there were external information about how to group mutations or if networks were much larger than considered here, than the strategy could be fruitful. A third limitation is that the model currently treats missing genotypes as simply absent. But if the genotypes are missing because they are not viable—something that will be especially common when mutations are deleterious - then the current approach is biased. Our model could be improved by treating inviable genotypes as having fitness censured by a lower boundary.

The methods and code presented here provide a framework for selecting among three basic landscape models. Sometimes, simple models are more useful than complex models when, for example, computational efficiency or mathematical simplicity are paramount. But the simplicity comes at a cost, of course. These models cannot explain patterns like sign epistasis (except in treating it as noise) and when they do fit data well, they fail to provide a mechanistic explanation of it. We know that in reality mutations manifest their effects on fitness through their phenotypic effects. We are enthusiastic about modeling efforts that delve into phenotypic dimensions, including, for example, extensions of Fisher's geometric model (e.g. [6], [8]), models built on metabolic principles (e.g. [48], [55]), and models linked to protein stability (e.g. [73], [74]). We argue that the value and insight from these more complex models is far more compelling when the models they aim to improve upon are not straw men. We see one of the main extension of this work, therefore, as addressing how the basic models should be compared to more complex models. The tools provided here, we hope, make this and related uses of these basic landscape models readily accessible.

\section{Acknowledgments}

This work was supported by the National Institutes of Health R01 GM076040. JTVL was supported by P20 GM104420 and computational resources were provided by P30 GM103324. CRM and HAW would like to note that this was the last work we did with Paul Joyce. Indeed, his enthusiasm for the stickbreaking model was our best strategy for getting him out of administrator mode and into math mode while he was dean.

\section{Appendix}

\section{Relative Distance to the Boundary (RDB)}

Recall that $r_{G}$ defined by equation (6) has the following form for the stickbreaking model

$$
r_{G}=\frac{d-\left(w_{G}-w_{w t}\right)}{d}=\prod_{i \in G}\left(1-u_{i}\right) .
$$

We obtain an expression for $r_{G}$ based on observable fitness effects independent of $d$. We do this by calculating the relative distance of genotype $G$ from $K$, the genotype containing all available mutations. If we replace $d$ with $w_{K}-w_{W t}$ in the left hand side of the above equation and using equation (3) we get 


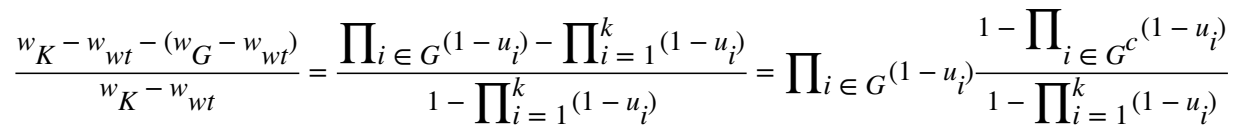

$$
\begin{aligned}
& =\prod_{i \in G^{\left(1-u_{i}\right)} \frac{{ }^{w} G^{c}-w_{w t}}{w_{K}-w_{w t}}}
\end{aligned}
$$

which implies

$$
r_{G}=\frac{w_{K}-w_{G}}{w_{G}-w_{w t}}=\prod_{i \in G}\left(1-u_{i}\right) .
$$

Equation (18) reveals that if one places genotype $G^{c}$ into the $G$ background one obtains fitness effect $w_{K}-w_{G}$, which under the stickbreaking model is smaller than the fitness gain produced by placing $G^{c}$ into the wildtype background $\left({ }_{G^{c}}-w_{w t}\right)$. Comparison of the two fitness effects produces the RDB for $G$.

Note that equation (18) only applies for a proper subset $G$ of $K$ and cannot be used to calculate to calculate the RDB for $K$. However, we can still obtain an expression for $r_{K}$ by applying equation (18) to the genotype containing the single mutation $j$ and the genotype containing all mutations but $j$, denoted by $j^{c}$.

$$
\frac{w_{K}-w_{j}}{w_{j}-w_{w t}}=1-u_{j}
$$

and

$$
\frac{w_{K}-w_{j}{ }^{c}}{w_{j}-w_{w t}}=\prod_{i \neq j}\left(1-u_{i}\right)
$$

implying that

$$
r_{K, j}=\left(\frac{w_{K}-w_{j}}{w_{j}{ }_{j}-w_{w t}}\right)\left(\frac{w_{K}-w_{j}{ }_{j}}{w_{j}-w_{w t}}\right)=\prod_{i=1}^{k}\left(1-u_{i}\right) .
$$

Note that $r_{K}=r_{K, j}$ for all $j$, but when we add noise to the mix, than the above will give us a set of estimates of $r_{K}$ for each $j$. 


\section{References}

1Smith J Maynard. Natural selection and the concept of a protein space. Nature. 1970; 225:563-564. [PubMed: 5411867]

2Gillespie JH. Molecular evolution over the mutational landscape. Evolution. 1984; 38(5):1116-1129. [PubMed: 28555784]

3Orr HA. The genetic theory of adaptation: A brief history. Nature Reviews Genetics. 2005; 6:119127.

4de Visser JAGM, Krug J. Empirical fitness landscapes and the predictability of evolution. Nature Review Genetics. 2014; 15(7):480-490.

5Fisher RA. The genetical theory of natural selection, Oxford University Press, Oxford (UK). 1930

6Martin G, Lenormand T. A general multivariate extension of Fisher's geometrical model and the distribution of mutation fitness effects across species. Evolution. 2006; 60(5):893-907. [PubMed: 16817531]

7Rokyta D, Joyce P, Caudle S, Miller C, Beisel C, Wichman H. Epistasis between beneficial mutations and the phenotype-to-fitness map for a ssDNA virus. PLoS Genetics. 2011; 7:e1002075. [PubMed: 21655079]

8Blanquart F, Bataillon T. Epistasis and the structure of fitness landscapes: are experimental fitness landscapes compatible with Fisher's geometric model? Genetics. 2016; 203(2):847-862. [PubMed: 27052568]

9Kondrashov FA, Kondrashov AS. Multidimensional epistasis and the disadvantage of sex. Proceedings of the National Academy of Sciences. 2001; 98(21):12089-12092. DOI: 10.1073/pnas. 211214298

10Otto SP. The evolutionary enigma of sex. The American Naturalist. 2009; 174(S1):S1-S14.

11 de Visser JAG, Park SC, Krug J. Exploring the effect of sex on empirical fitness landscapes. The American Naturalist. 2009; 174(S1):S15-S30.

12Orr HA. The population genetics of speciation: the evolution of hybrid incompatibilities. Genetics. 1995; 139:1805-1813. [PubMed: 7789779]

13 Gavrilets S. Fitness landscapes and the origin of species (MPB-41), Princeton University Press, Princeton, NJ. 2004

14Dettman JR, Sirjusingh C, Kohn LM, Anderson JB. Incipient speciation by divergent adaptation and antagonistic epistasis in yeast. Nature. 2007; 447(7144):585-588. [PubMed: 17538619]

15Gould SJ. Wonderful life: the Burgess Shale and the nature of history WW Norton \& Company; 1990

16Weinreich DM, Delaney NF, DePristo MA, Hartl DL. Darwinian evolution can follow only very few mutational paths to fitter proteins. Science. 2006; 312:111-114. [PubMed: 16601193]

17Lobkovsky AE, Wolf YI, Koonin EV. Predictability of evolutionary trajectories in fitness landscapes. PLoS Computational Biology. 2011; 7(12):e1002302. [PubMed: 22194675]

18Kryazhimskiy S, Rice DP, Jerison ER, Desai MM. Global epistasis makes adaptation predictable despite sequence-level stochasticity. Science. 2014; 344(6191):1519-1522. [PubMed: 24970088]

19Weinreich DM, Chao L. Rapid evolutionary escape by large populations from local fitness peaks is likely in nature. Evolution. 2005; 59(6):1175-1182. [PubMed: 16050095]

20Desai MM, Fisher DS, Murray AW. The speed of evolution and maintenance of variation in asexual populations. Current Biology. 2007; 17(5):385-394. [PubMed: 17331728]

21 Good BH, Rouzine IM, Balick DJ, Hallatschek O, Desai MM. Distribution of fixed beneficial mutations and the rate of adaptation in asexual populations. Proceedings of the National Academy of Sciences. 2012; 109(13):4950-4955.

22Otwinowski J, Plotkin JB. Inferring fitness landscapes by regression produces biased estimates of epistasis. Proceedings of the National Academy of Sciences. 2014; 111(22):E2301-E2309.

23Gillespie JH. The causes of molecular evolution Oxford University Press; New York: 1991

24de Vos MG, Poelwijk FJ, Battich N, Ndika JD, Tans SJ. Environmental dependence of genetic constraint. PLoS Genetics. 2013; 9(6):e1003580. [PubMed: 23825963] 
25Hietpas RT, Bank C, Jensen JD, Bolon DN. Shifting fitness landscapes in response to altered environments. Evolution. 2013; 67(12):3512-3522. [PubMed: 24299404]

26Caudle SB, Miller CR, Rokyta DR. Environment determines epistatic patterns for a ssDNA virus. Genetics. 2014; 196(1):267-279. [PubMed: 24214342]

27Kauffman S, Levin S. Towards a general theory of adaptive walks on rugged landscapes. Journal of Theoretical Biology. 1987; 128:11-45. [PubMed: 3431131]

28Perelson AS, Macken CA. Protein evolution on partially correlated landscapes. Proceedings of the National Academy of Sciences. 1995; 92(21):9657-9661.

29Orr HA. Theories of adaptation: what they do and don't say. Genetica. 2005; 123:3-13. [PubMed: 15881676]

30Rokyta DR, Beisel CJ, Joyce P. Properties of adaptive walks on uncorrelated landscapes under strong selection and weak mutation. Journal of Theoretical Biology. 2006; 243:114-120. [PubMed: 16859714]

31Park SC, Krug J. Clonal interference in large populations. Proceedings of the National Academy of Sciences. 2007; 104(46):18135-18140.

32Kryazhimskiy S, Tkačik G, Plotkin JB. The dynamics of adaptation on correlated fitness landscapes. Proceedings of the National Academy of Sciences. 2009; 106(44):18638-18643.

33Draghi JA, Plotkin JB. Selection biases the prevalence and type of epistasis along adaptive trajectories. Evolution. 2013; 67(11):3120-3131. [PubMed: 24151997]

34Murray BG. Population dynamics. JSTOR. 1979

35Bull J, Badgett M, Wichman HA, Huelsenbeck JP, Hillis DM, Gulati A, Ho C, Molineux I. Exceptional convergent evolution in a virus. Genetics. 1997; 147(4):1497-1507. [PubMed: 9409816]

36Andersson DI, Hughes D. Antibiotic resistance and its cost: is it possible to reverse resistance? Nature Reviews Microbiology. 2010; 8(4):260. [PubMed: 20208551]

37 Gerrish PJ, Lenski RE. The fate of competing beneficial mutations in an asexual population. Genetica. 1998; 102:127. [PubMed: 9720276]

38Sniegowski PD, Gerrish PJ. Beneficial mutations and the dynamics of adaptation in asexual populations. Philosophical Transactions of the Royal Society of London B: Biological Sciences. 2010; 365(1544):1255-1263. [PubMed: 20308101]

39Levy SF, Blundell JR, Venkataram S, Petrov DA, Fisher DS, Sherlock G. Quantitative evolutionary dynamics using high-resolution lineage tracking. Nature. 2015; 519(7542):181. [PubMed: 25731169]

40Walkiewicz K, Cardenas ASB, Sun C, Bacorn C, Saxer G, Shamoo Y. Small changes in enzyme function can lead to surprisingly large fitness effects during adaptive evolution of antibiotic resistance. Proceedings of the National Academy of Sciences. 2012; 109(52):21408-21413.

41Jacquier H, Birgy A, Le Nagard H, Mechulam Y, Schmitt E, Glodt J, Bercot B, Petit E, Poulain J, Barnaud G, et al. Capturing the mutational landscape of the beta-lactamase tem-1. Proceedings of the National Academy of Sciences. 2013; 110(32):13067-13072.

42Meini MR, Tomatis PE, Weinreich DM, Vila AJ. Quantitative description of a protein fitness landscape based on molecular features. Molecular Biology and Evolution. 2015; 32(7):1774-1787. [PubMed: 25767204]

43Lee YH, DSouza LM, Fox GE. Equally parsimonious pathways through an rna sequence space are not equally likely. Journal of Molecular Evolution. 1997; 45(3):278-284. [PubMed: 9302322]

44Aita T, Iwakura M, Husimi Y. A cross-section of the fitness landscape of dihydrofolate reductase. Protein Engineering. 2001; 14(9):633-638. [PubMed: 11707608]

45Lunzer M, Miller SP, Felsheim R, Dean AM. The biochemical architecture of an ancient adaptive landscape. Science. 2005; 310:499-501. [PubMed: 16239478]

46Lozovsky ER, Chookajorn T, Brown KM, Imwong M, Shaw PJ, Kamchonwongpaisan S, Neafsey DE, Weinreich DM, Hartl DL. Stepwise acquisition of pyrimethamine resistance in the malaria parasite. Proceedings of the National Academy of Sciences. 2009; 106(29):12025-12030. 
47da Silva J, Coetzer M, Nedellec R, Pastore C, Mosier DE. Fitness epistasis and constraints on adaptation in a human immunodeficiency virus type 1 protein region. Genetics. 2010; 185(1):293303. [PubMed: 20157005]

48Chou H, Chiu H, Delaney N, Segrè D, Marx C. Diminishing returns epistasis among beneficial mutations decelerates adaptation. Science. 2011; 332:1190-1192. [PubMed: 21636771]

49Khan AI, Dinh DM, Schneider D, Lenski RE, Cooper TF. Negative epistasis between beneficial mutations in an evolving bacterial population. Science. 2011; 332(6034):1193-1196. [PubMed: 21636772]

50Franke J, Klözer A, de Visser JAG, Krug J. Evolutionary accessibility of mutational pathways. PLoS Computational Biology. 2011; 7(8):e1002134. [PubMed: 21876664]

51Goulart CP, Mahmudi M, Crona KA, Jacobs SD, Kallmann M, Hall BG, Greene DC, Barlow M. Designing antibiotic cycling strategies by determining and understanding local adaptive landscapes. PloS One. 2013; 8(2):e56040. [PubMed: 23418506]

52Natarajan C, Inoguchi N, Weber RE, Fago A, Moriyama H, Storz JF. Epistasis among adaptive mutations in deer mouse hemoglobin. Science. 2013; 340(6138):1324-1327. [PubMed: 23766324]

53Tufts DM, Natarajan C, Revsbech IG, Projecto-Garcia J, Hoffmann FG, Weber RE, Fago A, Moriyama H, Storz JF. Epistasis constrains mutational pathways of hemoglobin adaptation in high-altitude pikas. Molecular Biology and Evolution. 2014:msu311.

54Sanjuán R, Moya A, Elena SF. The contribution of epistasis to the ar chitecture of fitness in an RNA virus. Proceedings of the National Academy of Sciences. 2004; 101(43):15376-15379.

55Bank C, Hietpas RT, Jensen JD, Bolon DN. A systematic survey of an intragenic epistatic landscape. Molecular Biology and Evolution. 2015; 32(1):229-238. [PubMed: 25371431]

56Bank C, Hietpas RT, Wong A, Bolon DN, Jensen JD. A Bayesian MCMC approach to assess the complete distribution of fitness effects of new mutations: uncovering the potential for adaptive walks in challenging environments. Genetics. 2014; 196(3):841-852. [PubMed: 24398421]

57Sarkisyan KS, Bolotin DA, Meer MV, Usmanova DR, Mishin AS, Sharonov GV, Ivankov DN, Bozhanova NG, Baranov MS, Soylemez O, et al. Local fitness landscape of the green fluorescent protein. Nature. 2016; 533(7603):397-401. [PubMed: 27193686]

58Steinberg B, Ostermeier M. Environmental changes bridge evolutionary valleys. Science Advances. 2016; 2(1):e1500921. [PubMed: 26844293]

59Brown KM, Costanzo MS, Xu W, Roy S, Lozovsky ER, Hartl DL. Compensatory mutations restore fitness during the evolution of dihydrofolate reductase. Molecular Biology and Evolution. 2010; 27(12):2682-2690. [PubMed: 20576759]

60Miller C, Joyce P, Wichman H. Mutational effects and population dynamics during viral adaptation challenge current models. Genetics. 2011; 187:185-202. [PubMed: 21041559]

61Szendro IG, Schenk MF, Franke J, Krug J, De Visser JAG. Quantitative analyses of empirical fitness landscapes. Journal of Statistical Mechanics: Theory and Experiment. 2013; 2013(01):P01005.

62Schenk MF, Szendro IG, Salverda ML, Krug J, de Visser JAG. Patterns of epistasis between beneficial mutations in an antibiotic resistance gene. Molecular Biology and Evolution. 2013; 30(8):1779-1787. [PubMed: 23676768]

63Nahum JR, Godfrey-Smith P, Harding BN, Marcus JH, Carlson-Stevermer J, Kerr B. A tortoise-hare pattern seen in adapting structured and unstructured populations suggests a rugged fitness landscape in bacteria. Proceedings of the National Academy of Sciences. 2015; 112(24):7530 7535.

64Martin G, Elena SF, Lenormand T. Distributions of epistasis in microbes fit predictions from a fitness landscape model. Nature Genetics. 2007; 39(4):555-560. [PubMed: 17369829]

65Pearson VM, Miller CR, Rokyta DR. The consistency of beneficial fitness effects of mutations across diverse genetic backgrounds. PloS One. 2012; 7(8):e43864. [PubMed: 22937113]

66Nagel AC, Joyce P, Wichman HA, Miller CR. Stickbreaking: a novel fitness landscape model that harbors epistasis and is consistent with commonly observed patterns of adaptive evolution. Genetics. 2012; 190(2):655-667. [PubMed: 22095084]

67Weinreich DM, Lan Y, Wylie CS, Heckendorn RB. Should evolutionary geneticists worry about higher-order epistasis? Current Opinion in Genetics \& Development. 2013; 23(6):700-707. [PubMed: 24290990] 
68Sailer ZR, Harms MJ. Detecting high-order epistasis in nonlinear genotype-phenotype maps. Genetics. 2017; 205(3):1079-1088. [PubMed: 28100592]

69McCandlish DM, Otwinowski J, Plotkin JB. Detecting epistasis from an ensemble of adapting populations. Evolution. 2015; 69(9):2359-2370. [PubMed: 26194030]

70R Core TeamR: A Language and Environment for Statistical Computing R Foundation for Statistical Computing; Vienna, Austria: 2015 URL https://www.R-project.org/

71Burns CC, Shaw J, Campagnoli R, Jorba J, Vincent A, Quay J, Kew O. Modulation of poliovirus replicative fitness in HeLa cells by de-optimization of synonymous codon usage in the capsid region. Journal of Virology. 2006; 80(7):3259-3272. [PubMed: 16537593]

72Orr HA, Day T. The population genetics of adaptation on correlated fitness landscapes: the block model. Evolution. 2006; 60(6):1113-1124. [PubMed: 16892963]

73Serohijos AW, Shakhnovich EI. Merging molecular mechanism and evolution: theory and computation at the interface of biophysics and evolutionary population genetics. Current Opinion in Structural Biology. 2014; 26:84-91. [PubMed: 24952216]

74Rodrigues JV, Bershtein S, Li A, Lozovsky ER, Hartl DL, Shakhnovich EI. Biophysical principles predict fitness landscapes of drug resistance. Proceedings of the National Academy of Sciences. 2016:201601441. 

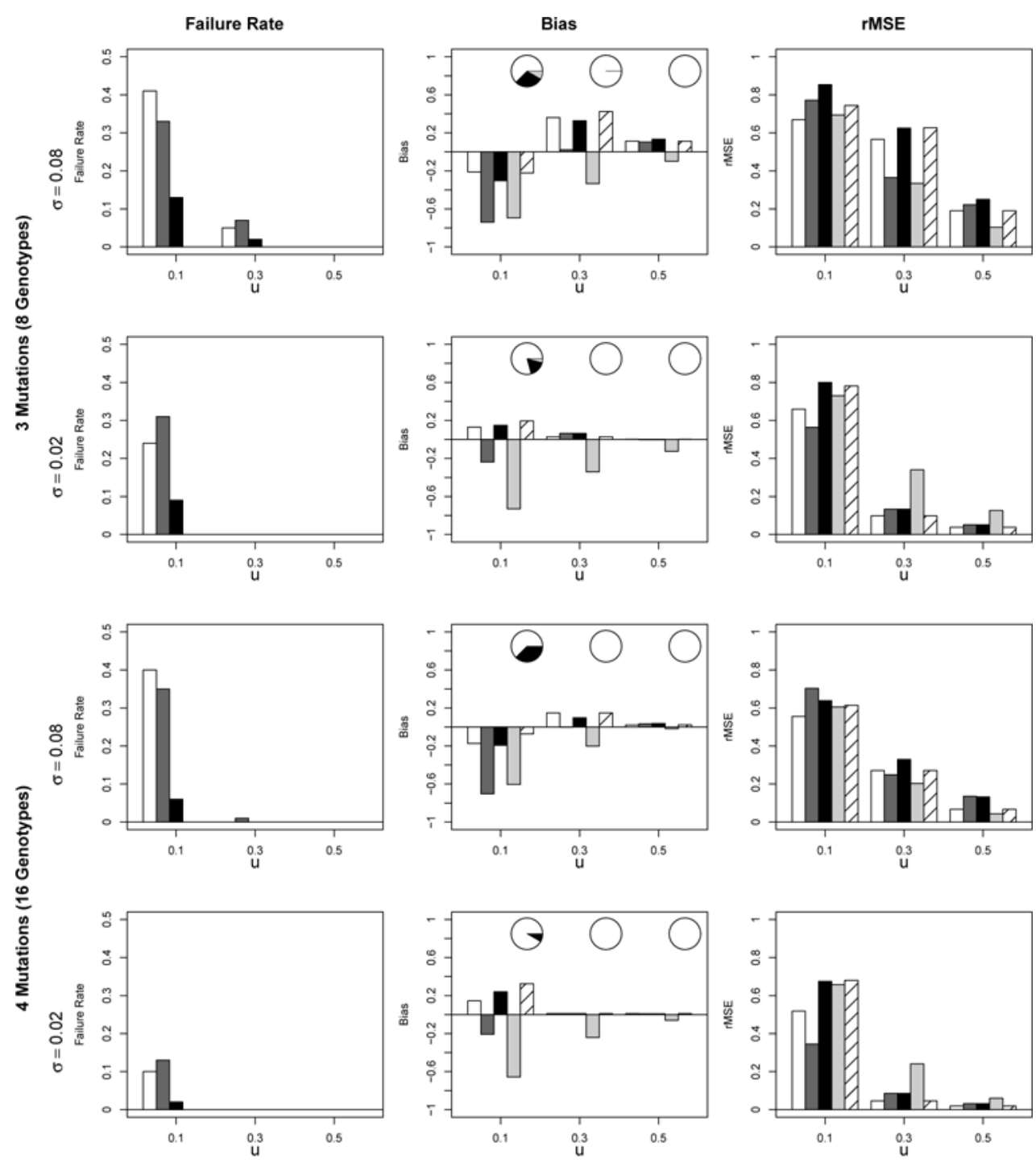

\section{$\square \hat{\mathrm{d}}_{\mathrm{MLE}} \square \hat{\mathrm{d}}_{\mathrm{RDB} \mathrm{B}_{\mathrm{al}}} \square \hat{\mathrm{d}}_{\mathrm{RDB}} \square \hat{\mathrm{d}}_{\mathrm{Max}} \square \hat{\mathrm{d}}_{\mathrm{Hybrid}}$}

Figure 1.

Failure rate (left column), bias (central) and root mean squared error (rMSE; right) for several estimators of the distance to the stickbreaking boundary, $d$ (inset legend). Number of mutations and $\sigma$ indicated to left of panels; coefficient size, $u$, on x-axis. Inset pie-charts show proportion of hybrid estimates based on MLE, RDB and Max estimators. Results based on 100 simulations per condition. 

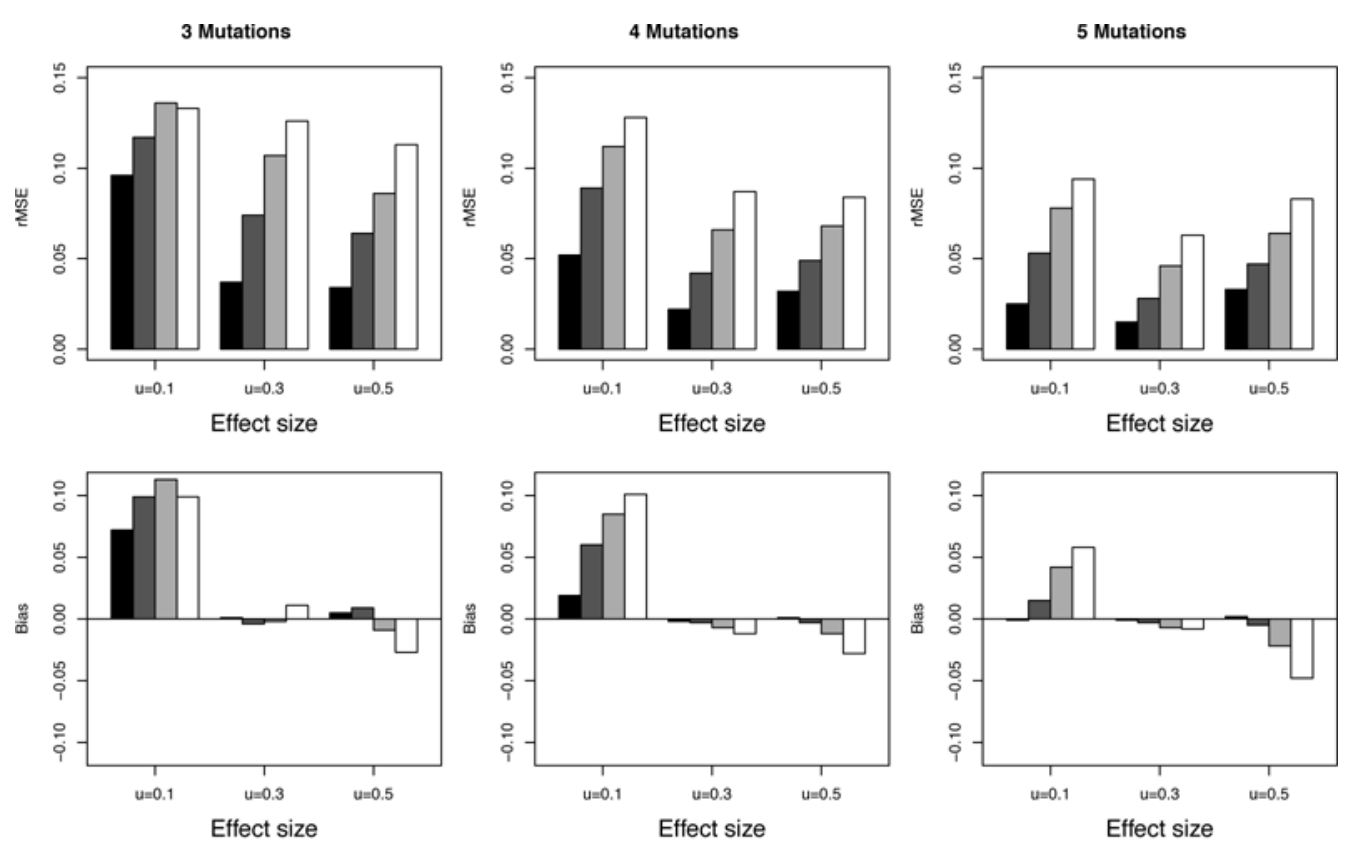

$\sigma$

$0.02 \square 0.04 \quad \square \quad 0.06 \square 0.08$

Figure 2.

Root mean squared error (rMSE; top row of panels) and bias (bottom) of estimates of stickbreaking coefficients $(u)$ as a function of number of mutations (columns of panels), effect size (x-axis), and $\sigma$ (shaded bars, see legend at bottom). All estimates are based on the hybrid method of estimating $d$. Based on $d=1$ and 1000 simulations per condition. 

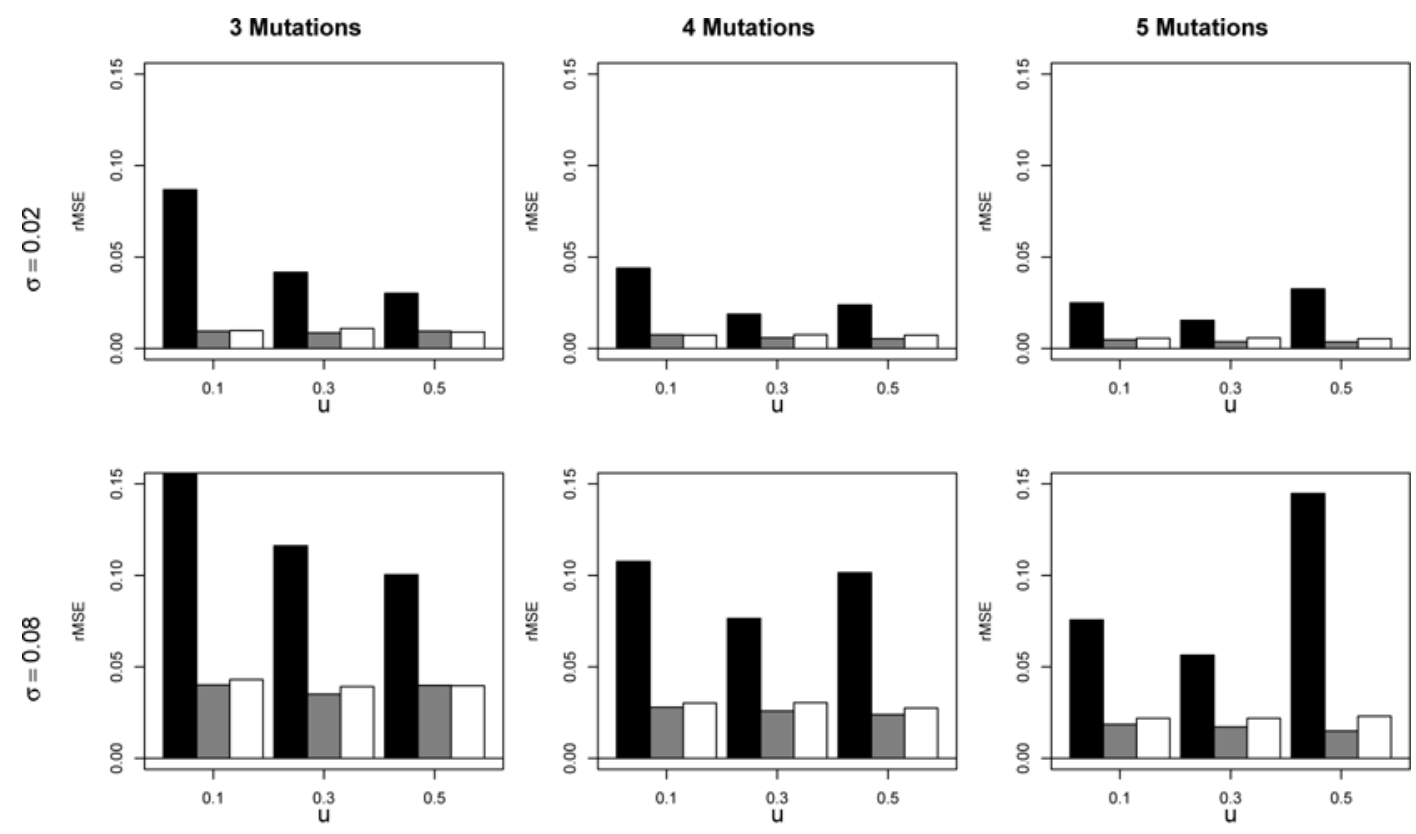

- Stickbreaking $\square$ Multiplicative $\square$ Additive

Figure 3.

Root mean squared error (rMSE) of selection coefficients under all three models as a function of number of mutations (columns of panels), $\sigma$ (top row 0.02, bottom 0.08), effect size (x-axis), and model (shaded bars, see legend at bottom). Based on 1000 simulations per condition. 
3 Mutations
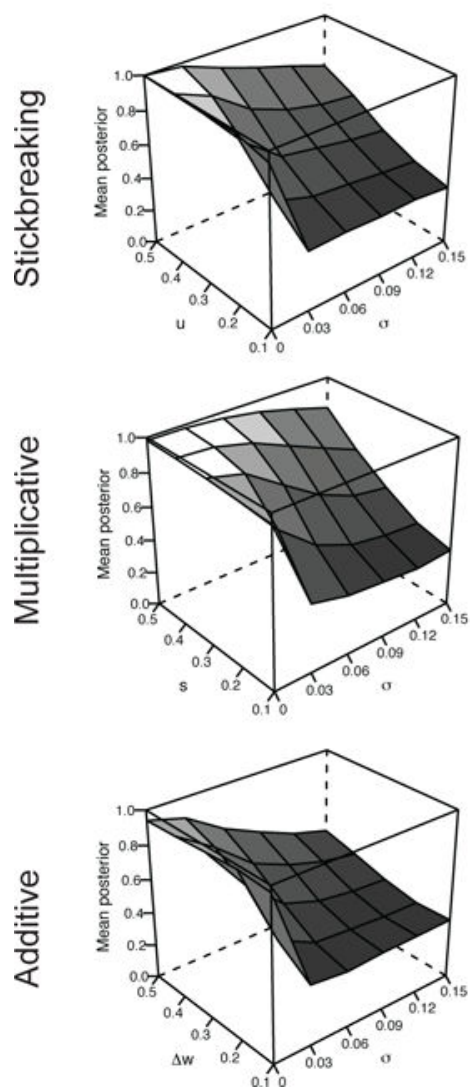

4 Mutations
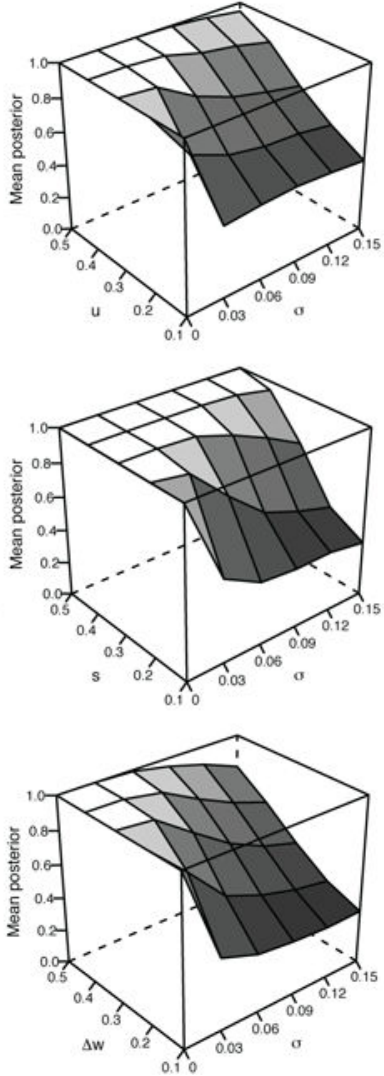

5 Mutations
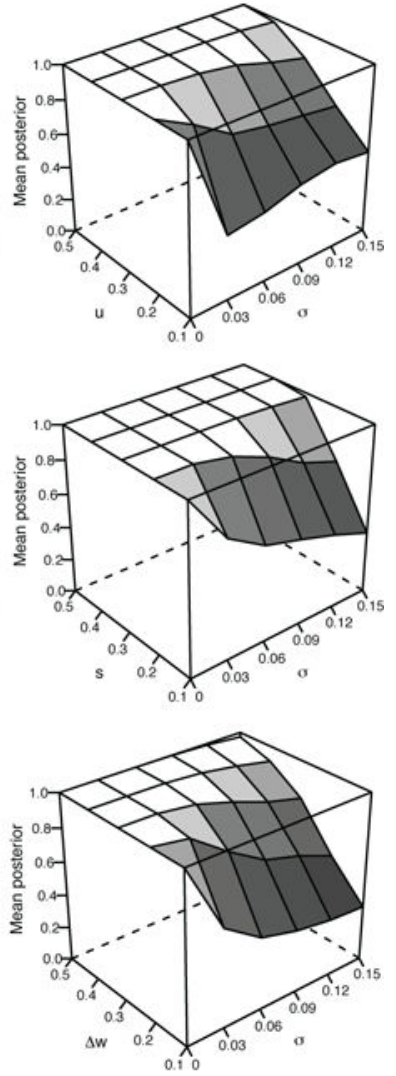

Figure 4.

Mean posterior probability of the true model (z-axis in each plot) as a function of model (panel rows), number of mutations (panel columns), $\sigma$ (x-axis), and effect size ( $u, s$, and $\Delta W$, $\mathrm{y}$-axis). Shaded white are regions with mean posterior probability $\geq 0.95$. 
A

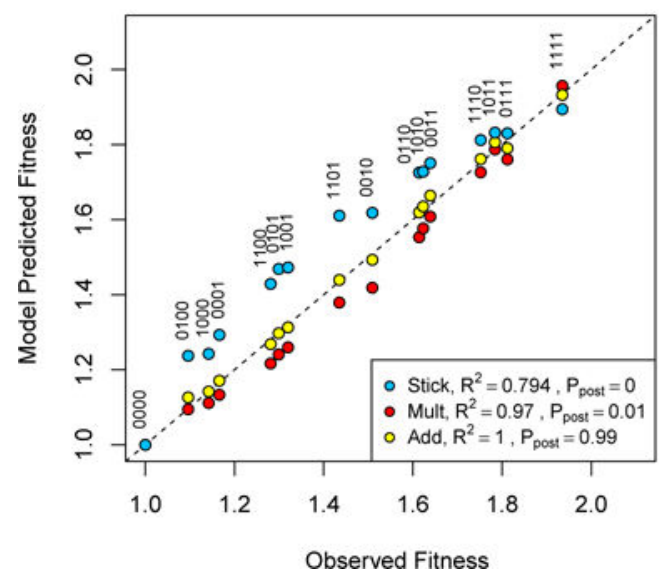

B

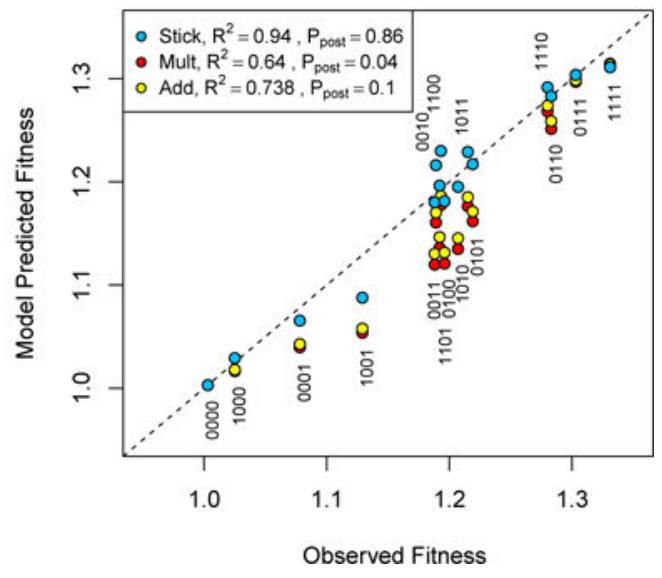

Figure 5.

Observed versus model predicted fitness for two empirical datasets. In both cases, the inset legend indicates the model, $R^{2}$, and posterior probability of model $\left(P_{\text {post }}\right)$. Each genotype in a dataset corresponds to a trio of vertically aligned circles (one per model) with the binary string indicating absence (0) and presence (1) of the individual mutations. (A) In the dataset from Chou et al. (2011) from Methylobacterium, the additive model fits very well and receives virtually all the posterior probability. The binary strings correspond to mutations fghA, pnt $A B, g s h A$, and $G B$ in that order. In addition to the $R^{2}$ values shown in legend, the P-scores strongly favored the additive model: $P_{\text {stick }}=-24.7, P_{\text {mult }}=-20.3$, and $P_{\text {add }}=-4.9$. (B) In the Khan et al. (2011) data from $E$ coli, the stickbreaking model fits best and receives $86 \%$ of the posterior probability, although the additive model cannot be rejected. As discussed in text, one outlier mutation $(p y k F)$ was removed from the data before analysis. The binary strings correspond to mutations $\triangle r b s$, topA, spoT, and $g \operatorname{lm} U S$ in that order. The P-scores also contribute support to the stickbreaking model: $P_{\text {stick }}=-0.59, P_{\text {mult }}=-15.2$, and $P_{a d d}=-12.2$. 


\section{A}

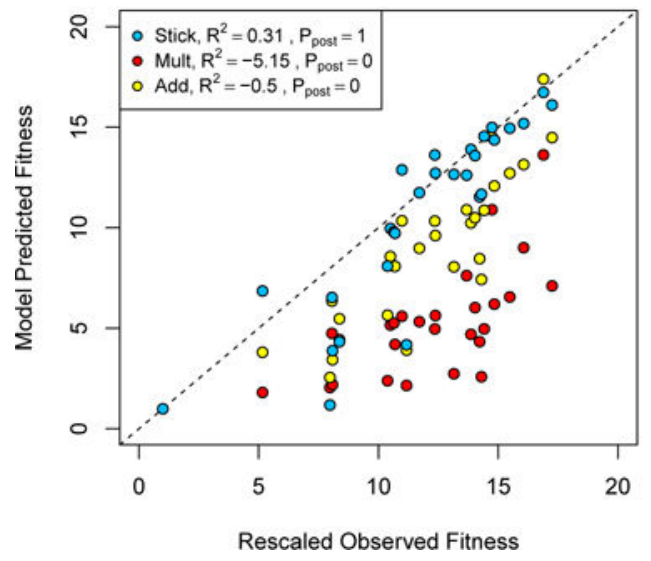

B

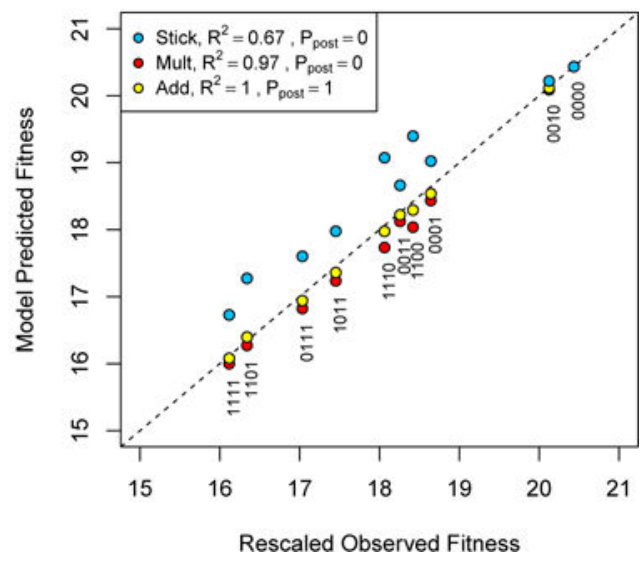

Figure 6.

Observed versus model predicted fitness for subnetwork datasets. In both cases, the inset legend indicates the model, $R^{2}$, and posterior probability of model $\left(P_{\text {post }}\right)$. (A) In the dataset from Caudle et al. ([26]) from the bacteriophage ID11, the stickbreaking model fits the data much better than the other two, although several of the single mutations to the left have large errors. The $\mathrm{P}$ scores for the three models strongly contributed to the high posterior probability associated with stickbreaking: $P_{\text {stick }}=-11.5, P_{\text {mult }}=-29.8$, and $P_{\text {add }}=-29.9$. (B) Combining synonymously recoded blocks of the poliovirus by Burns et al. ([71]) follows the additive model better than the other two. Each genotype in a dataset corresponds to a trio of vertically aligned circles (one per model) with the binary strings indicating absence (0) and presence (1) of the individual mutations (or mutated blocks). The P-scores also contributed to the additive posterior probability: $P_{\text {stick }}=-12.3, P_{\text {mult }}=-3.3$, and $P_{\text {add }}=-2.5$ 


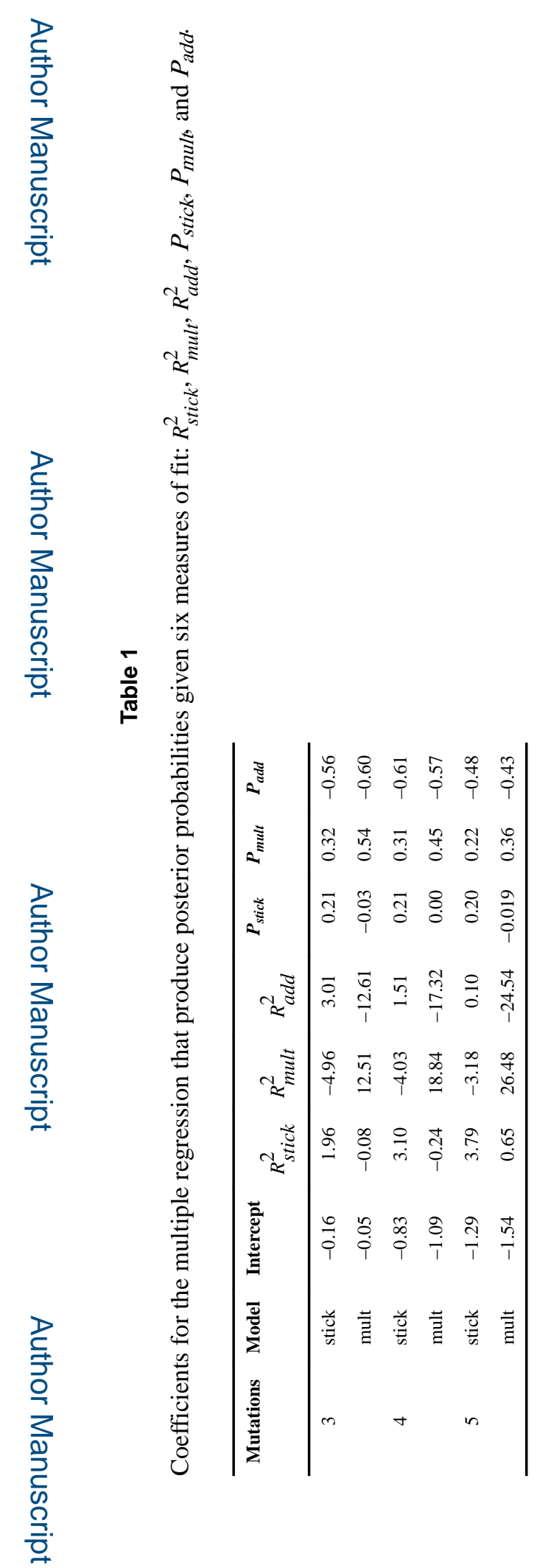

Theor Popul Biol. Author manuscript; available in PMC 2019 July 01. 